\title{
Tratamiento Laser-Lok® en implantes dentales. A propósito de un caso clínico
}

\section{Laser-Lok® treatment on implants dental. About a clinical case}

\author{
M. Blázquez-Hinarejos, K. Gómez-Izquierdo**, A. Estrugo-Devesa***, \\ R. Ayuso-Montero***, J. López-López****.
}

\begin{abstract}
RESUMEN
Uno de los retos más importantes, hoy en día, en implantología oral es evitar la pérdida ósea alrededor de los implantes oseointegrados y funcionales con el paso del tiempo. Una de las superficies que se ha desarrollado en los últimos años es la resultante de la microtexturación mediante láser. Diversos estudios han demostrado la adhesión del tejido conectivo a las superficies tratadas con láser en la zona cervical de los implantes oseointegrados, consiguiendo de este modo un sellado biológico a este nivel. Revisamos lo publicado sobre este tema y presentamos un caso clínico de inserción de un implante unitario con tratamiento láser en su zona cervical desde su colocación hasta la restauración final, donde se observa la buena integración de los tejidos y el éxito del caso a largo plazo.
\end{abstract}

PALABRAS CLAVE: Implantes, láser, cervical, tejidos blandos, oseointegración.

\section{ABSTRACT}

There are certainly situations where the standard implants can not be placed or suppose a risk for the crestal bone maintenance. The narrow implants have been used for a wide variety of indications, stablishing scientifically-based treatment protocols with excellent long-term results.In the present study we present a narrative review of narrow implants highlighting their indications and limitations, and a case report where narrow implants have been sh it is evident that narrow implants are an alternative when rehabilitate edentulous spaces with low available bone in the horizontal direction or reduced mesio-distal spaces in aesthetic areas.

KEY WORDS: Implants, laser, cervical, soft tissue, osseointegration.

Fecha de recepción: 7 de enero de 2020

Fecha de aceptación: 11 de enero de 2020

* Máster en Medicina, Cirugía e Implantología Oral. Facultad de Medicina y Ciencias de la Salud, Universidad de Barcelona.

* * Máster en Medicina, Cirugía e Implantología Oral. Facultad de Medicina y Ciencias de la Salud, Universidad de Barcelona. // Departamento de Odontoestomatología. Facultad de Medicina y Ciencias de la Salud (Odontología), Universidad de Barcelona

*** Departamento de Odontoestomatología. Facultad de Medicina y Ciencias de la Salud (Odontología), Universidad de Barcelona // Oral Health and Masticatory System Group, Institut d'Investigació Biomédica de Bellvitge (IDIBELL, Bellvitge Institute of Biomedical Research), L'Hospitalet de Llobregat, Barcelona, España.

**** Departamento de Odontoestomatología. Facultad de Medicina y Ciencias de la Salud (Odontología), Universidad de Barcelona // Oral Health and Masticatory System Group, Institut d'Investigació Biomédica de Bellvitge (IDIBELL, Bellvitge Institute of Biomedical Research), L'Hospitalet de Llobregat, Barcelona, España // Director Facultativo y Responsable del Área Médico Quirúrgica del Hospital Odontológico Universidad de Barcelona (HOUB). L'Hospitalet de Llobregat, Barcelona, España. 


\section{INTRODUCCIÓN}

La rehabilitación oral mediante implantes dentales oseointegrados es una técnica utilizada desde hace varias décadas por profesionales de todo el mundo con tasas de éxito elevadas ${ }^{(1)}$. Este hecho hace que sea una de las principales opciones de tratamiento con las que contamos a la hora de rehabilitar a pacientes parcial o totalmente edéntulos ${ }^{(2,3)}$.

No obstante, si bien los implantes dentales tienen una larga durabilidad, pueden sufrir pérdida de oseointegración por reabsorción ósea, que puede provocar el fracaso del implante. Desde su inicio se empezaron a observar las primeras pérdidas de oseointegración y se ha estudiado en profundidad las diferentes variables que influyen en la pérdida ósea con el fin de evitarla. Así, conocemos que las principales causas de la pérdida del implante dental son la calidad ósea pobre de la zona quirúrgica, la periodontitis crónica, las enfermedades sistémicas, el hábito tabáquico, edad avanzada, la localización del implante, los hábitos parafuncionales, la pérdida de la integración del implante y la restauración con una prótesis inadecuada ${ }^{(4-6)}$.

Es por lo anterior que uno de los retos más importantes hoy en día en el campo de la implantología oral, es prevenir la pérdida ósea alrededor de los implantes oseointegrados y funcionales con el paso del tiempo. Desde hace unos años se sabe que los dos factores principales de la pérdida ósea periimplantaria son la sobrecarga del implante y la proliferación bacteriana hacia el interior de la unión hueso-implante ${ }^{(7-10)}$.

La enfermedad periimplantaria se inicia por un desequilibrio entre la carga bacteriana y las defensas del huésped ${ }^{(11)}$. La mucosa oral protege los tejidos periodontales frente a bacterias y otros estímulos dañinos, pero cuando se coloca un implante, esta barrera se rompe y se crea una discontinuidad, favoreciendo la penetración de bacterias por este margen. Además el epitelio oral tiene una capacidad inferior para sellar el implante que para sellar un diente natural ${ }^{(12)}$.

Con el fin de intentar solucionar este problema se han diseñado diferentes tipos de implantes y pilares, así como modificaciones en la técnica de su colocación, con el propósito de lograr que exista una mejor unión de los tejidos blandos al implante y minimizar

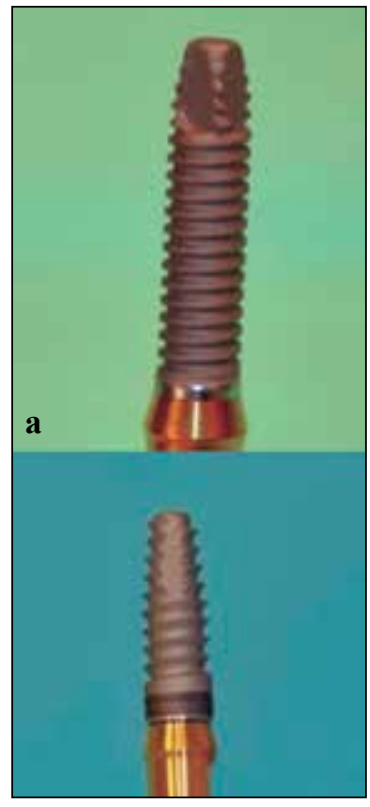

Figura 1. a) Implante con cuello mecanizado. B). Implante tratado con láser en la zona cervical. la pérdida ósea ${ }^{(13)}$. Uno de los métodos que se ha desarrollado con este objetivo es conseguir una superficie de implante que permite un correcto sellado periférico de los tejidos blandos en su zona cervical; de este modo, se forma una barrera fisiológica que evita el desarrollo de la enfermedad periimplantaria y su consecuente pérdida ósea $^{(14)}$.

Para ello existe un tratamiento en la zona cervical del implante, que se crea mediante espiras similares a las existentes en el cuerpo del implante, pero con una separación entre ellas de menor tamaño, también denominadas microespiras ${ }^{(15)}$. En un trabajo en animales, se compararon implantes de cuello mecanizado con implantes con microespiras e implantes con cuello tratado mediante láser ${ }^{(16)}$ (Fig. 1). Tras su estudio histológico se llegó a la conclusión de que en los implantes tratados con láser en su zona cervical y en los que tenían microespiras la integración del tejido conectivo era mejor, siendo algo superior en los implantes con microsurcos creados mediante láser.

La microtexturización mediante láser es una de las superficies que se ha desarrollado intensamente en los últimos años (Fig. 2), y que se utiliza tanto en implantes como en pilares implantológicos. Nuestro grupo publicó una revisión sistemática donde se evaluaron diferentes tipos de tratamiento de superficie en pilares implantológicos en lugar de en implantes ${ }^{(17)}$, que tuviesen repercusión en el sellado biológico entre la encía y el pilar. Se encontró que el tratamiento de superficie basado en la creación de microsurcos mediante láser pueden aportar un beneficio en la unión del tejido conectivo al pilar ${ }^{(17)}$.

Por otro lado, otros estudios centrados en la modificación de superficie de los implantes en lugar de los 


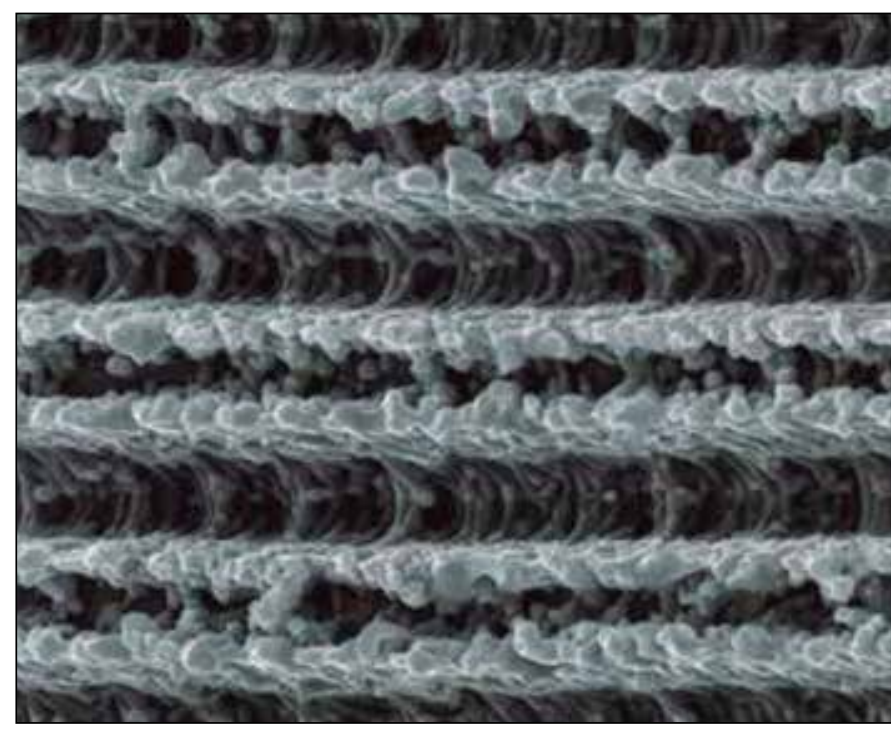

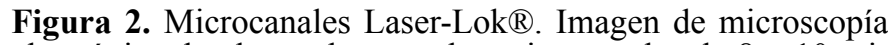
electrónica donde se observan los microcanales de 8 y 10 micras. (Imágen cedida por Biohorizons ${ }^{\circledR}$ ).

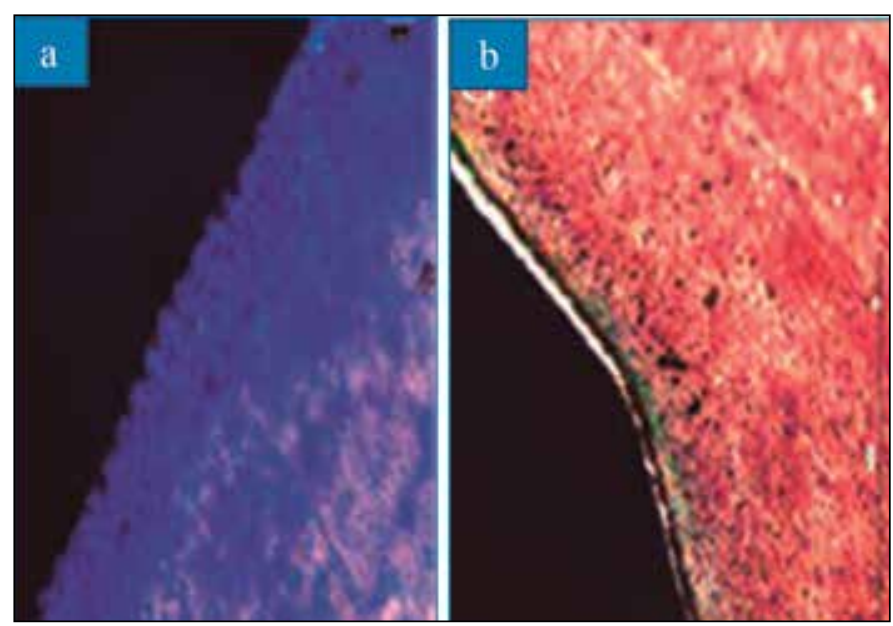

Figura 3. a) Pilar con tratamiento láser. b) Pilar de superficie lisa. Fotografías tomadas de las muestras histológicas del estudio clínico de Blázquez-Hinarejos et al ${ }^{\cdot(23)}$.

pilares, han demostrado la adhesión del tejido conectivo a las superficies tratadas con láser en la zona cervical de los implantes oseointegrados, consiguiéndose de este modo un sellado biológico a este nivel ${ }^{(18-22)}$.

En este contexto, Weiner et al.(18) estudiaron por primera vez en modelo animal el efecto que producía sobre el hueso, los cuellos microtexturizados con láser en implantes, así como en el tejido conectivo y en las células epiteliales circundantes. Llegaron a la conclusión de que este tratamiento estimulaba la adhesión ósea y del tejido blando, facilitando el desarrollo de un ancho biológico correcto. Por otro lado, Nevins et al. ${ }^{(19)}$ analizaron muestras histológicas humanas, y demostraron la eficacia de los microsurcos creados mediante láser en los cuellos de los implantes, para conseguir una conexión directa del tejido conectivo al implante. De este modo se inhibe la migración apical del epitelio de unión. Pecora et al. ${ }^{(20)}$ observaron radiográficamente una pérdida ósea de 0,59 $\mathrm{mm}$ en implantes tratados con láser, mientras que en implantes control tuvieron una pérdida ósea de $1,94 \mathrm{~mm}$. Posteriormente, Shapoff et al. ${ }^{(21)}$ observaron una pérdida ósea mínima en 49 implantes con cuello tratados con láser de $0,44 \mathrm{~mm}$ a los 2 años y de 0,46 $\mathrm{mm}$ a los 3 años, demostrando un correcto sellado biológico entre las fibras de tejido conectivo y el implante. En este mismo tema, un estudio posterior de Botos et al. ${ }^{(22)}$ demostró, que además de encontrar menor pérdida ósea alrededor de implantes con cuellos tratados con láser, también las profundidades de sondaje eran inferiores en estos implantes en comparación a implantes convencionales.

Tras estudiar estas evidencias en implantes, los autores publicamos un estudio clínico en humanos más reciente, donde evaluamos las diferencias histológicas entre los tejidos blandos alrededor de pilares lisos y pilares tratados con láser en su zona cervical ${ }^{(23)}$. En el estudio encontramos diferencias estadísticamente significativas entre el porcentaje de unión existente entre el grupo de pilares tratados con láser y el grupo de pilares sin tratamiento; y llegamos a la conclusión principal de que el tejido conectivo tiene una mejor adhesión a los pilares tratados con láser en comparación a los pilares lisos. El nivel de adhesión de los tejidos blandos pudo comprobarse mediante la observación de muestras histológicas con microscopio óptico (Fig. 3).

Toda esta bibliografía mencionada apoya la teoría de que el tratamiento láser, tanto en implantes como en los pilares, puede ayudar a una mejor inserción de los tejidos y por consiguiente a una menor pérdida ósea en el tiempo. Clínicamente esta modificación de la superficie de la zona cervical del implante nos ayuda a prolongar el éxito de nuestras restauraciones a largo plazo.

Con el fin de mostrar el beneficio del tratamiento láser en la zona cervical de los implantes, presentamos un caso clínico donde puede evidenciarse el correcto sellado de los tejidos blandos, así como la ausencia de reabsorción ósea alrededor de este tipo de implantes. 


\section{CASO CLÍNICO}

1.- Se trata de una paciente de 48 años que acude al servicio del Máster de Medicina, Cirurgia i Implantologia Oral de la Facultat d'Odontologia de la Universitat de Barcelona. La paciente tras un accidente de tráfico había sufrido la pérdida del incisivo central superior derecho y como medida temporal era portadora de una prótesis provisional removible (Fig. 4). La paciente no padecía ninguna enfermedad sistémica, no tomaba medicación de forma habitual y no presentaba ningún hábito tóxico.

2.- Se procede a realizar modelos de estudio para realizar el posterior encerado diagnóstico. Se solicita una ortopantomografía (Fig. 5A). Además se realiza un mapeo de 3 en $3 \mathrm{~mm}$ de la zona, previa anestesia infiltrativa, midiendo mediante sonda periodontal y compás el grosor gingival hasta el reborde alveolar tanto en palatino como en vestibular, así como el grosor óseo (Fig. 5B).

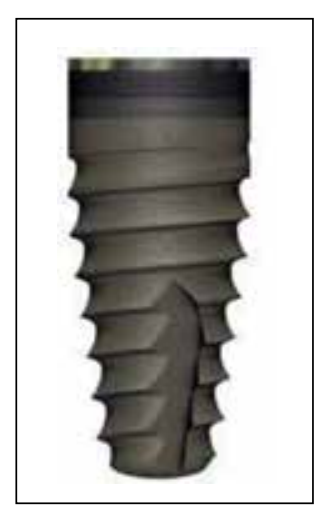

Figura 6. Implante Biohorizons tapered internal Laser-Lok ${ }^{\circledR}$. En el caso clínico se utilizó uno de $12 \mathrm{~mm}$ de longitud y diámetro de 4,6 mm (Imagen cedida por Biohorizons $\left.{ }^{\circledR}\right)$.
3.- Analizado el caso se decide realizar implante con carga y provisionalización diferida. Se utiliza un implante Biohorizons $®$ tapered Laser-Lok® (Fig. 6).

Es un implante de conexión interna, con cuerpo de forma cónica y en su zona cervical dispone de una zona tratada mediante láser, con el cual se crean unos microsurcos o microcanales Laser-Lok®. Estos microsurcos consisten en una serie de canales circunferenciales del tamaño celular, son extremadamente consistentes y tienen el tamaño óptimo para adherir y organizar tanto a los fibroblastos como a los osteoblastos. La microestructura Laser-Lok® también incluye una nanoestructura repetitiva que maximiza el área de superficie y permite que los pseudópodos y las microfibrillas de colágeno se entrelacen con la superficie Laser-Lok®.

4.- Previo a la intervención quirúrgica de inserción del implante dental se prescribe a la paciente la cobertura antibiótica de $2 \mathrm{~g}$ de amoxicilina 1 hora antes de la intervención y $750 \mathrm{mg}$ repartidos en 3 dosis diarias los 5 días posteriores ${ }^{(24)}$. Como tratamiento coadyuvante domiciliario, se prescribieron enjuagues con solución

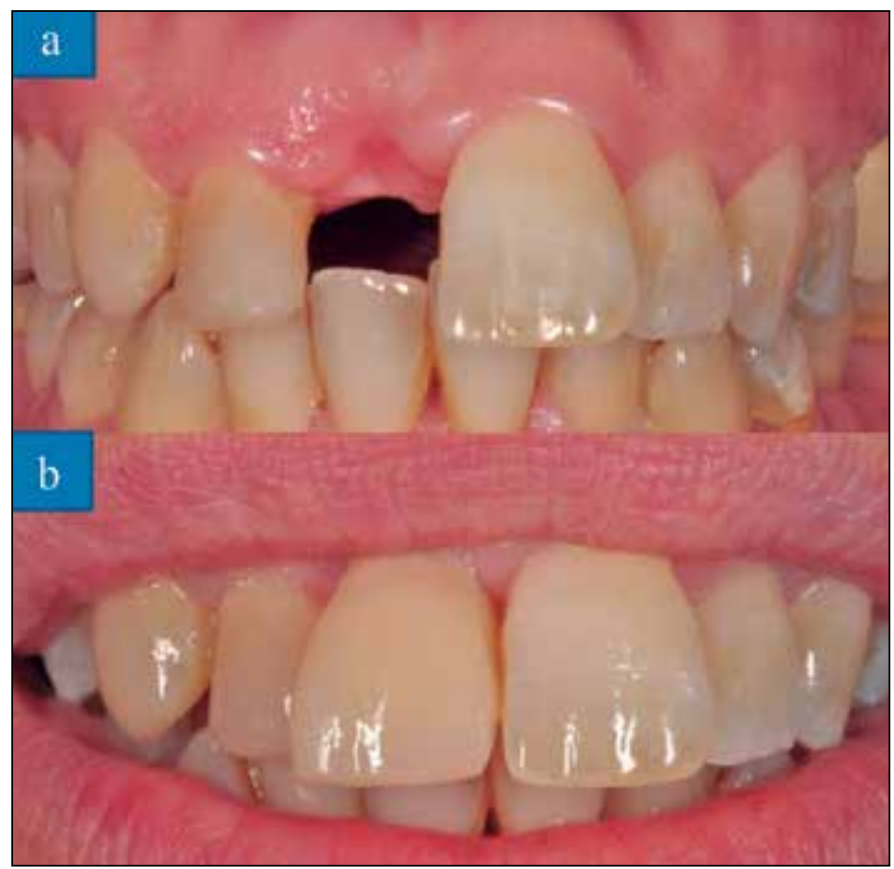

Figura 4. a) Fotografías realizadas en la primera visita. b) Prótesis provisional.

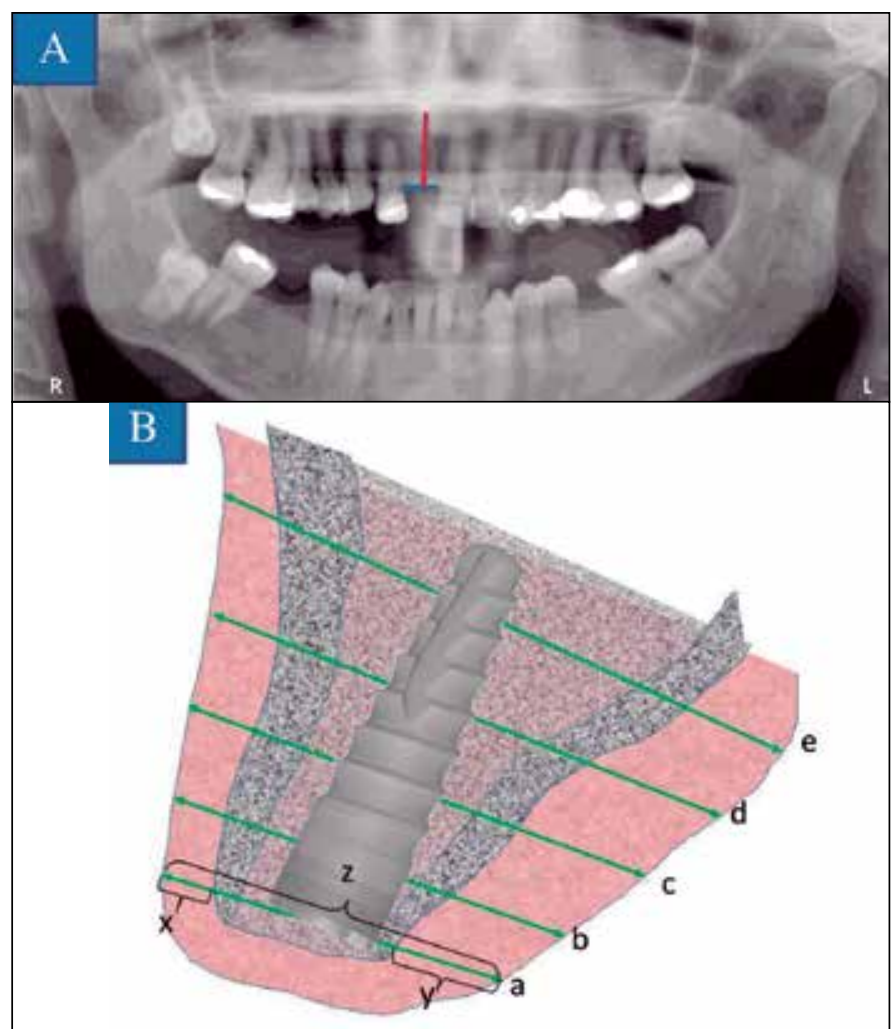

Figura 5. A) Ortopantomografia inicial. B) Simulación del alveolo mediante mapeo [cada $3 \mathrm{~mm}$ ] con sonda y con compas. Mediciones realizadas desde a hasta e con $3 \mathrm{~mm}$ de distancia entre ellas. Nomenclatura $=[\mathrm{x} / \mathrm{z} / \mathrm{y}]$, donde $\mathrm{x}$ es grosor de encía vestibular; $\mathrm{y}$ : medición total $\mathrm{y} \mathrm{z}$ : medición grosor de encía palatina. $a=[2 / 14 / 3] ; b=[2 / 15 / 5] ; c=[2 / 15 / 6]$; $\mathrm{d}=[3 / 20 / 8]$ y e $=[5 / 25 / 9]$. 
de clorhexidina $0,12 \%$ durante los 7 primeros días y al 0,05 los restantes 7 días y con una posología de 3 veces al día.

Se realiza una incisión sulcular discretamente palatinizada con una descarga a distal del 12, respetando la papila y un despegamiento de espesor total. El implante se inserta según el protocolo recomendado por la casa comercial y la sutura empleada fue de 6.0 (Monofilamento de poliéter poliuretánico no absorbible, Normon®, Barcelona, España). Su diámetro reducido permitió reducir el acúmulo de placa alrededor de la herida quirúrgica.

5.- A los 14 días de la intervención quirúrgica se realizó una visita postoperatoria para la retirada de la sutura y el control de la herida (Fig. 7).

6.- Posteriormente se realizaron controles pasados 30 y 60 días desde la intervención quirúrgica. A los 90 días post inserción del implante se realizó otra visita de control, donde se obtuvo una ortopantomografía y se procedió a provisionalizar el implante (Fig. 8). De esta forma se pretende preservar al máximo y modelar en la medida de lo posible los tejidos blandos circundantes al implante. Para ello se confecciona una prótesis provisional atornillada mediante un pilar de PEEK (Polyetheretherketone).

7.- Al mes y con el fin de reproducir la anatomía de los tejidos blandos lo más exacta posible se realiza la impresión del implante utilizando la corona provisional. Ya que se había obtenido un margen gingival correcto, se hace una copia en resina de este margen, reproduciendo la zona gingival de la corona provisional y trasladándolo en resina a un pilar de impresión (Fig. 9). Finalmente, una vez realizadas las diferentes pruebas se coloca una corona definitiva sobre el implante en posición 11, confeccionada en cerámica e. max Press de Ivoclar Vivadent®, Barcelona, España (Fig. 10).

8.- Seguimiento del caso. Una vez colocada la prótesis definitiva, la paciente sigue acudiendo al Hospital Odontológico a visitas de control semestrales durante los 2 primeros años. En estas visitas se realiza control radiográfico y pruebas de sondaje. No se evidencia ni inflamación ni sangrado al sondaje y la profundidad es de unos $2 \mathrm{~mm}$ en todo el contorno del margen gingival. Radiográficamente no se observa pérdida ósea marginal alrededor del implante.

Una vez transcurridos estos 24 meses, la paciente no

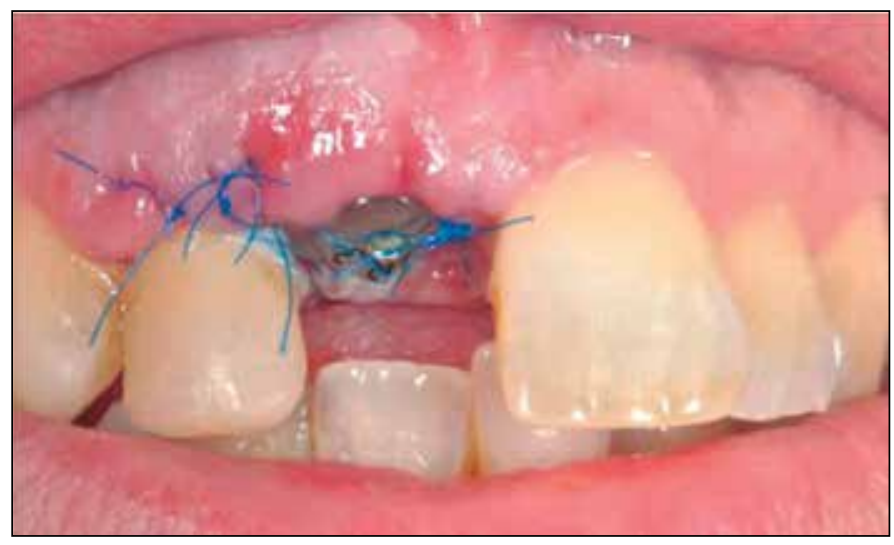

Figura 7. Control postquirúrgico a los 7 días.

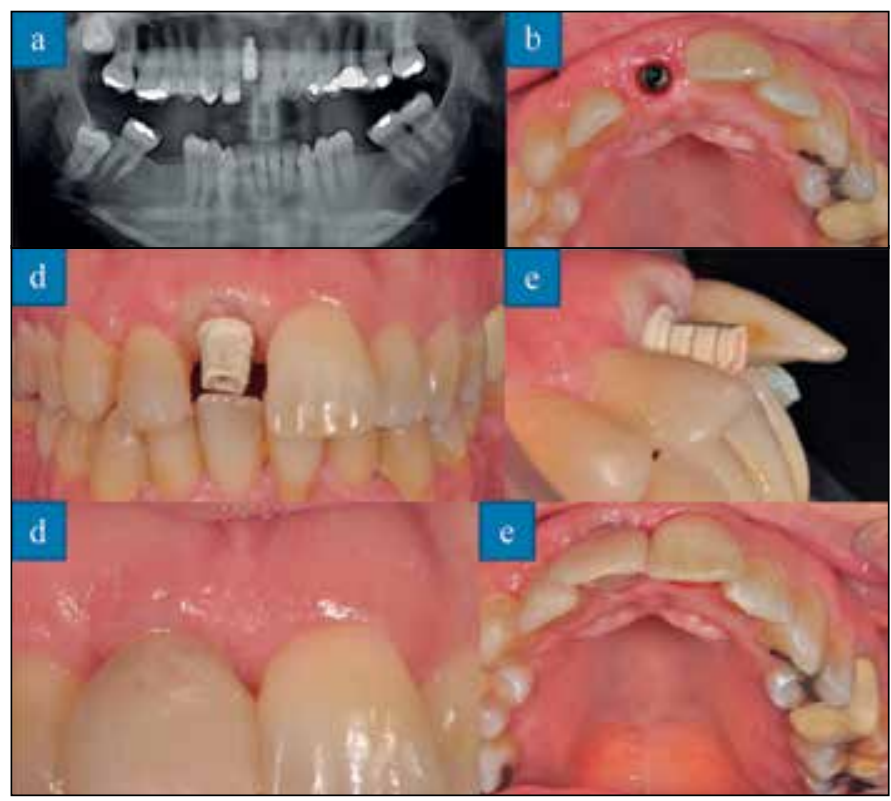

Figura 8. a y b) Ortopantomografía de control e implante sin tapón de cierre; b y c) Confección de corona provisional mediante pilar PEEK; d y e) Control tras 30 días con prótesis provisional.

acude a las citas anuales y en un control a los 6 años de finalizado el caso (acude debido a otro problema en su cavidad oral), se constata la buena evolución y el mantenimiento correcto los tejidos blandos circundantes (Fig.11).

\section{DISCUSIÓN}

Este caso clínico nos permite mostrar la óptima respuesta de los tejidos blandos al tratamiento láser en la zona cervical del implante dental. Diferentes estudios clínicos en humanos ${ }^{(17,18)}$ han comprobado los beneficios de los microsurcos creados con láser en los implantes dentales, ya sea mejorando la integración de los tejidos blandos como minimizando la pérdida 


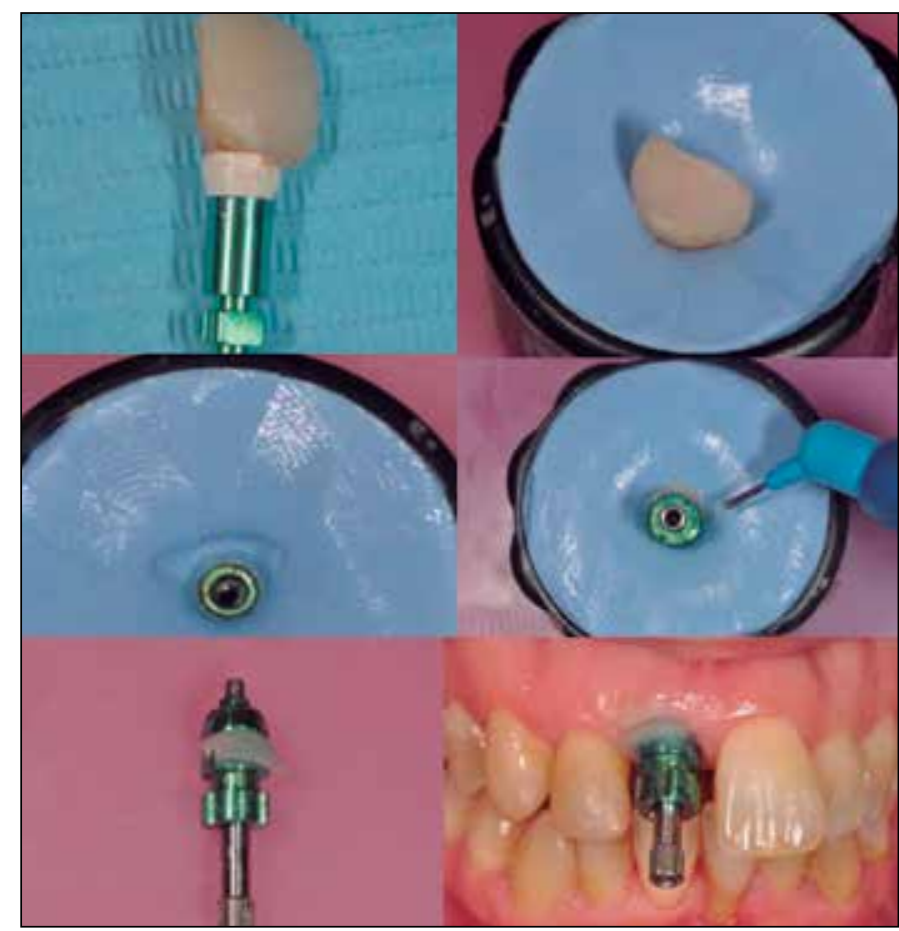

Figura 9. Pasos para obtener una impresión sobre el implante copiando los tejidos blandos mediante la corona provisional atornillada.

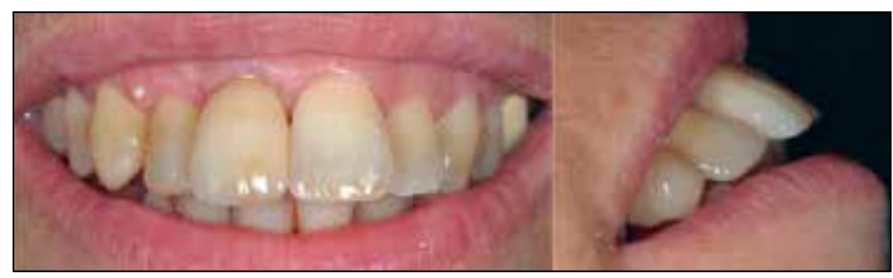

Figura 10. Colocación de la prótesis definitiva, en la que se observa la buena adaptación de los tejidos blandos.

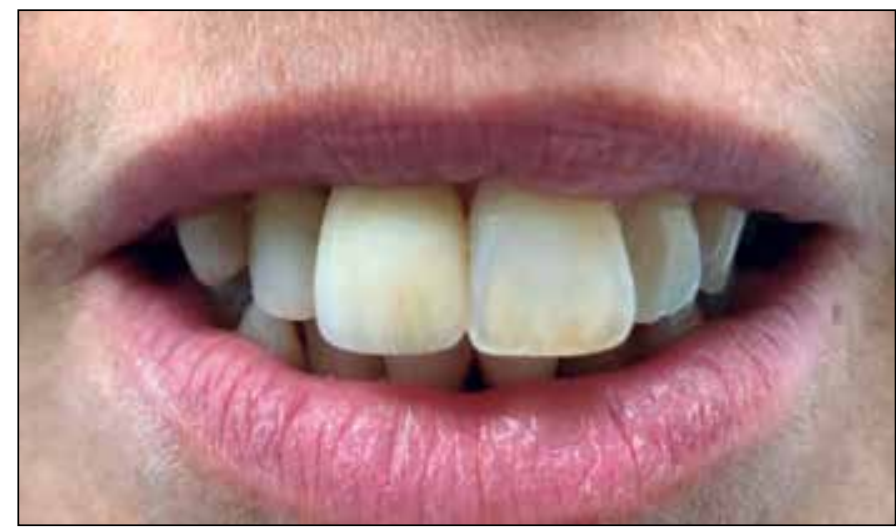

Figura 11. Control clínico a los 6 años.

ósea tanto en sentido vertical como horizontal.

En un estudio in vitro(25) en el que se comparaba la morfología y la proliferación celular sobre superficies tratadas con láser, de titanio y de zirconio se demos- tró que la morfología celular observada mediante microscopía electrónica era sustancialmente diferente en las superficies tratadas con láser en comparación al resto de superficies. Los autores observaron que la mayor parte de las células epiteliales del grupo Laser-Lok® eran fusiformes y con procesos parecidos a pseudópodos y las células redondas se encontraban en una proporción menor. Por otro lado, en el tejido gingival circundante a los pilares de zirconio observaron mayoritariamente células redondas. En el grupo de pilares de titanio mecanizado observaron células redondas y fusiformes en la misma proporción, aunque las células fusiformes eran menos alargadas que en el grupo Laser-Lok®.

Los autores concluyeron que en cuanto a la viabilidad celular, el tejido gingival correspondiente a las superficies Laser-Lok® tuvo una mayor proliferación celular. Además, la cantidad de fibronectina existente fue significativamente superior en tejido gingival integrado a los pilares Laser-Lok® en comparación a los pilares de titanio lisos sin tratamiento de superficie.

Estos hallazgos revelan que la morfología celular es muy importante para determinar la afinidad de las células por una determinada superficie. De hecho, las células con morfología fusiforme más longitudinal pueden tener una mayor integración a las superficies, debido a sus pseudópodos citoplasmáticos en comparación a las células de componente más circular ${ }^{(25)}$.

Nosotros publicamos un estudio clínico(17) en el que comparamos las diferencias histológicas en humanos entre los pilares sin tratamiento de superficie y los pilares con tratamiento láser, donde observamos y calculamos las diferencias de adhesión entre ambas superficies. Se observó que en los pilares con tratamiento láser frecuentemente se establecía un contacto óseo cercano en la superficie del pilar en su aspecto crestal.

El estudio cuantificó la cantidad de tejido conectivo subepitelial en contacto con la superficie del pilar y se observó que había una mayor integración del tejido conectivo a las superficies Laser-Lok $®$ en comparación a los pilares sin tratamiento de superficie. Estos hallazgos suponen un beneficio clínico en cuanto al mantenimiento de los tejidos blandos a largo plazo.

\section{CONCLUSIONES}

El tratamiento mediante láser en la zona cervical de los 
implantes dentales puede ser una muy buena opción para preservar el sellado biológico de los tejidos. Al existir un firme sellado de los tejidos blandos se impide la proliferación bacteriana hacia la zona de contacto entre hueso e implante y este hecho se traduce en una menor pérdida de hueso y en una tasa de éxito superior de este tipo de implantes a largo plazo.

\section{REFERENCIAS BIBLIOGRÁFICAS}

1. Misch CE. (2008) Contemporary Implant Dentistry, $3^{a}$ Edición. Editorial Mosby, St Louis (Missouri), p. 621-635.

2. Adell $R$, Eriksson $B$, Lekholm U, Brånemark PI, Jemt $T$. Long-term follow-up study of osseointegrated implants in the treatment of totally edentulous jaws. Int J Oral Maxillofac Implants 1990; 5(4):347-59.

3. Roos-Jasanker AM, Lindhal C, Renvert H, Renvertb S. Nine to fourteen year follow-up of implant treatment. Part I: implant loss and associations to various factors. Journal of Periodontology 2006; 33, 28389.

4. Alssadi G, Quiryen M, Komerek A, Van Steenberghe D. Impact of local and systemic factors on the incidence of oral implant failure, up to abutment connection. Journal of Clinical Periodontology 2007; 34: 610.

5. Alssadi G, Quiryen M, Komerek A, Van Steenberghe D. Impact of local and systemic factors on the incidence of late oral implant loss. Clinical Oral Implants Research 2008; 19: 670-6.

6. Alssadi G, Quiryen M, Michiles K. Impact of local and systemic factors on the incidence of implant failures, up to abutment connection with modified surface oral implants. Journal of Clinical Periodontology 2008; 35: 51.

7. Esposito M, Ekestubbe A, Grondahl K. Radiological evaluation of marginal bone loss at tooth surfaces facing single Brånemark implants. Clinical Oral Implants Research 1993; 4: 151-7.

8. Esposito M, Grusovin MG, Chew YS, Coulthard P, Worthington HV. One-stage versus two-stage implant placement: a Cochrane systematic review of randomised controlled clinical trials. European Journal of Oral Implantology 2009; 2: 91-9.

9. Isidor F. Loss of osseointegration caused by occlusal load of oral implants. A clinical and radiographic study in monkeys. Clinical Oral Implants Research 1996; 7:143-52.

10. Pontoriero R, Tonelli MP, Carnevale G, Mombelli A, Nyman SR, Lang NP. Experimentally induced peri-implant mucositis. A clinical study in humans. Clinical Oral Implants Research 1994; 5: 254-9.

11. Valente NA, Andreana S. Peri-implant disease: what we know and what we need to know. Journal of Periodontal $\mathcal{E}$ Implant Science 2016; 46: 136

12. Atsuta I, Ayukawa Y, Kondo R, Oshiro W, Matsuura Y, Furuhashi A. Soft tissue sealing around dental implants based on histological interpretation. Journal of Prosthodontic Research 2016; 60: 3-11.

13. Huang HL, Chang CH, Hsu JT, Fallgatter AM, Ko CC. Comparison of implant body designs and threaded designs of dental implants: A 3-dimensional finite ele- ment analysis. The International Journal of Oral $\&$ Maxillofacial
Implants 2007; 22: 551-62.

14. Moon IS, Berglundh T, Abrahamsson I, Linder E, Lindhe J. The barrier between the keratinized mucosa and the dental implant. An experimental study in the dog. Journal of Clinical Periodontology 1999; 26: 658-63.

15. Frenkel SR, Simon J, Alexander H, Dennis M, Ricci JL. Osseointegration on metallic implant surfaces: effects of microgeometry and growth factor treatment. J Biomed Mater Res 2002; 63:706-13.

16. Hanna E, Moon K, In-Ho C, Dong-Hoo H. Marginal tissue response to different implant neck design. The Journal of Korean Academy of Prosthodontics 2008; 6: 602-9.

17. Blázquez-Hinarejos $M$, Ayuso-Montero R, Jané-Salas $E$, López-López J. Influence of surface modified dental implant abutments on connective tissue attachment: A systematic review. Archives of Oral Biology 2017; 80: 185-92.

18. Weiner S, Simon J, Ehrenberg DS, Zweig B, Ricci JL. The effects of laser microtextured collars upon crestal bone levels of dental implants. Implant Dentistry 2008; 17: 217-28.

19. Nevins M, Nevins ML, Camelo M, Boyesen JL, Kim DM. Human histologic evidence of a connective tissue attachment to a dental implant. The International Journal of Periodontics $\mathcal{E}$ Restorative Dentistry 2008; 28: 111-21.

20. Pecora GE, Ceccarelli R, Bonelli M, Alexander H, Ricci JL. Clinical evaluation of laser microtexturing for soft tissue and bone attachment to dental implants. Implant Dentistry 2009; 18: 57-66.

21. Shapoff CA, Lahey B, Wasserlauf PA, Kim DM. Radiographic analysis of crestal bone levels around Laser-Lok collar dental implants. The International Journal of Periodontics $\mathcal{E}$ Restorative Dentistry 2010; 30: 129-37.

22. Botos S, Yousef H, Zweig B, Flinton R, Weiner S. The effects of laser microtexturing of the dental implant collar on crestal bone levels and peri-implant health. The Inte Journal of Oral E Maxillofacial Implants 2011; 26: 4.

23. Blázquez-Hinarejos M, Ayuso-Montero R, Álvarez-López JM, Manzanares-Céspedes MC, López-López J. Histological differences in the adherence of connective tissue to laser-treated abutments and standard abutments for dental implants. An experimental pilot study in humans. Med Oral Patol Oral Cir Bucal. 2017 Nov; 22(6): e774-9.

24. Caiazzo A, Casavecchia P, Barone A, Brugnami F. A pilot study to determine the effectiveness of different amoxicillin regimens in implant surgery. Journal of Oral Implantology 2011; 37: 691-6.

25. Esfahanizadeh N, Motalebi S, Daneshparvar N, Akhoundi N, Bonakdar S. Morphology, proliferation, and gene expression of gingival fibroblasts on Laser-Lok, titanium, and zirconia surfaces. Lasers in Medical Science 2016; 31: 863-73.

\section{DIRECCIÓN DE CORRESPONDENCIA:}

\section{Raúl Ayuso Montero}

Facultad de Medicina y Ciencias de la Salud (Odontología), Universidad de Barcelona Campus Universitario de Bellvitge.

Pabellón de Gobierno

C/ Feixa LLarga s/n 08907

L'Hospitalet de Llobregat Barcelona - España raulayuso@ub.edu 